\title{
Application of 630-nm and 850-nm Light-emitting Diodes and Microcurrent to Accelerate Collagen and Elastin Deposition in Porcine Skin
}

\author{
Tae-Rin Kwon ${ }^{1}$ \\ Dong Wook Moon' \\ Jungwook Kim \\ Hyoung Jun Kim ${ }^{1}$ \\ Seong Jae Lee ${ }^{1}$ \\ Yunhee $\operatorname{Han}^{1}$ \\ Hee Won Dan ${ }^{1}$ \\ Sang Hoon $\mathrm{Chi}^{1}$ \\ Hwan Mo Seong ${ }^{1}$ \\ Hee Jung Kim² \\ Guei-Sam Lim² \\ Jungkwan Lee ${ }^{1}$
}

${ }^{1}$ Home Entertainment Company, LG Electronics, Seoul, Korea

${ }^{2}$ Advanced R\&BD Center, CTO, LG Electronics, Seoul, Korea
Received May 24, 2021

Accepted May 27, 2021

Correspondence
Tae-Rin Kwon
Home Entertainment Company, LG Electronics,
222 LG-ro, Jinwi-myeon, Pyeongtaek 17709, Korea
Tel.: +82-31-686-4676
Fax: +82-31-610-5134
E-mail: kwon_taerinlahotmail.com
(C) Korean Society for Laser Medicine and Surgery
(c) This is an open access article distributed under the
terms of the Creative Commons Attribution Non-
Commercial License (http://creativecommons.org/
licenses/by-nc/4.0) which permits unrestricted non-
commercial use, distribution, and reproduction in any
medium, provided the original work is properly cited.

\author{
Background and Objectives \\ Skin aging is reportedly associated with regulation in collagen and elastin \\ synthesis. This study investigated the potential of combining light- \\ emitting diode (LED) treatments using a 630-nm and 850-nm LED with \\ simultaneous microcurrent application.
}

\section{Materials and Methods \\ The dorsal skin of female pigs was treated with a home-use device. We examined the treatment effects using photography, thermocamera, microscopic pathology, and histological examination to determine the mechanism of action, efficacy, and safety of the procedure. A histological observation was performed using hematoxylin and eosin, Masson's trichrome, Victoria blue, and immunohistochemical staining. We also used the Sircol soluble collagen and elastin assay kit to measure the amounts of collagen and elastin in the porcine back skin tissue after 2 and 6 weeks.}

\section{Results}

Evaluation by visual inspection and devices showed no skin damage or heat-induced injury at the treatment site. Histological staining revealed that accurate treatment of the targeted dermis layer effectively enhanced collagen and elastin deposition. Collagen type I, a protein defined by immunohistochemical staining, was overexpressed in the early stages of weeks 2 and 6 . Combined therapy findings showed the superior capability of the 630-nm and 850-nm LED procedures to induce collagen; in contrast, elastin induction was more pronounced after microcurrent treatments.

\section{Conclusion}

The home-use LED device, comprising a combination of $630-\mathrm{nm}$ and 850-nm LEDs and microcurrent, is safe and can be used as an adjunctive treatment for self-administered facial rejuvenation.

\section{Key words}

Microcurrent; Low-level light therapy; Collagen; Elastin; Combination 


\section{INTRODUCTION}

With the introduction of new cosmetic techniques, the demand for non-invasive, long-lasting treatments to reduce facial wrinkles and relaxation has increased dramatically over the past decades. ${ }^{1}$ Aging is a functional, structural, and biochemical change that occurs across the cells and organs of the body. ${ }^{2}$ In human skin, hormone secretion decreases over time, and the function and activity of immune cells decrease, resulting in decreased biosynthesis of biological proteins. This results from endogenous aging and externally contaminated air, drugs, and photoaging caused by ultraviolet rays. In sundamaged skin, collagen synthesis decreases as matrix metalloproteinase (MMP) increases. ${ }^{3}$ Therefore, as a strategy to prevent or treat medical signs of skin aging, collagen deficiency can be relieved through MMP reduction and new collagen synthesis. It has been reported that the use of various light-emitting diode (LED) light sources that emit light corresponding to wavelengths in the visible and near-infrared regions can enhance skin collagen synthesis and simultaneously suppress the increase in MMP. ${ }^{4}$ After irradiating the red LED light source through various experiments, a remarkable increase in collagen synthesis was reported. ${ }^{5}$

In addition, a microcurrent leads to a weak current of less than $1000 \mu \mathrm{A}$. Approximately $40 \mu \mathrm{A}$ to $60 \mu \mathrm{A}$ of 'bioelectricity' flows into the human body. ${ }^{6}$ Bioelectricity is a chemical reaction in the body caused by the constant exchange of various ions such as $\mathrm{Na}+\mathrm{K}+, \mathrm{Ca} 2+$, and $\mathrm{Cl}-$ through the cell membrane, which refers to the generation of minute electricity in this process as the materials carrying the electrodes are reversed. Bioelectricity, which occurs in the basic process of metabolism in the human body, is connected to the activation, growth, regeneration, and healing processes of cells, including signal transmission, muscle contraction, secretion of substances, and stimulus reception. ${ }^{7}$ However, performance and safety evaluation are essential to confirm the efficacy of various variables generated while delivering complex energy and efficacy when used in combination.

In the current study, we examined the efficacy and safety of a home-use device in improving skin regeneration using a pig model by using the devices twice a week for the two months. Pre-clinical efficacy was evaluated through investigator assessments and instrumental analysis for the objective measurement of elastin and collagen content.

First-generation home appliances that emit lowintensity red/infrared or bipolar radiofrequency have been shown to produce minor improvements in wrinkles and/ or skin texture due to the penetration of energy into the surface area. ${ }^{8}$ Laser-based home devices have recently been approved by the FDA for the treatment of periorbital wrinkles; nevertheless, they have limitations because they cannot treat large areas, depend on the skin type, and are associated with pain.

This study establishes that the use of EWN1 wearable devices in combination with eye patches can lead to significant improvements in facial skin condition and rejuvenation after 2 and 6 weeks of use. Instrumental assessments showed significant improvements in skin collagen content, firmness, and elasticity throughout the 6-week study period. Interestingly, the greatest improvement first appeared after 2 weeks of use and gradually increased in effect and statistical significance at 6 weeks. We confirm that this dose-dependent improvement is due in part to the physiology of energy-based collagen remodeling. Data on performance is one of the data required to be submitted for review of technical documents, etc., and in particular, corresponds to data proving the effectiveness of medical devices used for diagnosis or treatment. If the performance of the device cannot be confirmed through an in vitro test, the performance of the device must be verified using test data for animals. In this case, refer to the thesis and literature for the standards and test methods of animal test data.

The results of this study indicate that the EWN1 multisource home-use device offers a non-invasive, effective, safe, and painless treatment option for self-administered skin rejuvenation. To our knowledge, this is the first time that two energy-based home use facial rejuvenation devices have been tested in a controlled pre-clinical study using both visual and instrumental assessments for improved skin rejuvenation. The EWN1 wearable device was demonstrated to be safe for facial locations with minimal downtime and unwanted side effects.

This study was designed to determine whether patients could safely and effectively apply a novel wearable LEDs and microcurrent device intended for home use to improve skin rejuvenation by stimulating collagen and elastin.

\section{MATERIALS AND METHODS}

\section{Animals and experimental design}

In this study, female pigs ( $n=2,4$ sites) obtained from the closed barrier unit at CRONEX (Hwasung, Korea) were used. They were housed individually under controlled environmental conditions (temperature, $18-22^{\circ} \mathrm{C}$; relative air humidity, 30-70\%; 15 air changes/h; 12:12-h light- 
dark cycle). After each pig's general condition and weight (range, 40-50 kg) were confirmed, they were placed under preliminary anesthesia by intramuscular injection using a 3-ml mixture of zoletil 50 (tiletamine hydrochloride plus zolazepam hydrochloride, Virbac S.A, France) and Rompun (xylazine hydrochloride, Bayer, Leverkusen, Germanyl at a ratio of 6:4. Each subject was then moved to an operating room, laid on the operating table, and 2 $\mathrm{ml}$ of the same mixture was administered via intravenous injection. The airway was secured using a laryngoscope, and an intubation tube (8.5 Fr) was inserted. Terel solution lisoflurane; Piramal Critical Care, Inc., Bethlehem, PA, USAl and oxygen was mixed at a 2:1 ratio, and the mixture was used as inhalation anesthesia before treating the dorsal skin areas using the EWN1 home-use device (LG Electronics, Seoul, Korea) (Fig. 1). 'Eye Care' is a type of collagen care in which LG PraL's exclusive 630-nm and 850 -nm LED for the eye area is added to the microcurrent, and it intensively manages the skin around the eyes.

Treatment using 630-nm and 850-nm LED only, microcurrent only, and combination (Basic Care Mode 19 min operation) level 1 and 3 (LED 25 mW Spectrometer, Integration Range: 550-710 nm, Wavelength 630 nm/LED 25 mW Spectrometer, Integration Range: $790-910 \mathrm{~nm}$, Wavelength $850 \mathrm{~nm} /$ Microcurrent DC $\pm 7.5 \mathrm{~V}$, 1 level : 100-

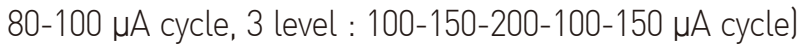

was performed twice a weeks for 2 and 6 weeks. The pigs were followed for 42 days ( 6 weeks) post-treatment before being humanely sacrificed. All procedures involving animals were conducted in accordance with the guidelines provided by the Care and Use of Laboratory Animals of the National Institutes of Health. The protocol was approved by the Institutional Animal Care and Use Committee of CRONEX, Republic of Korea (Approval Number:2020-09001).

\section{Observation and examination}

After anesthetizing Yorkshire pigs, the back area was epilated to apply EWN1 device. EWN1 treatment was performed according to each condition. The skin conditions of the treated and untreated areas (patch only) were observed to evaluate the skin irritation caused by the use of the EWN1 device. Immediately after treatment (day 0), 1 d after treatment (day 1), 7 days (day 7), 14 days (2 weeks), and 42 days ( 6 weeks), visual evaluation was performed by the research director.

The skin tissue was biopsied at weeks 2 and 6 and used for the performance evaluation. Skin surface measurements of the temperature distribution following treatment were performed on porcine back skin using an infrared thermography system (TVS-200EX; Avionics, Tokyo, Japan). Standardized flash photography of the treated and
A

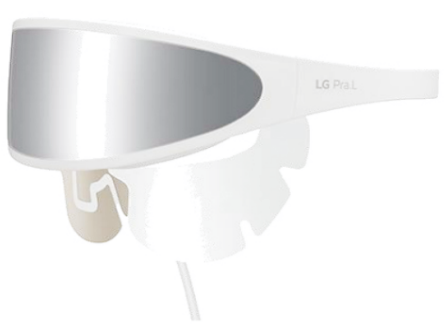

B

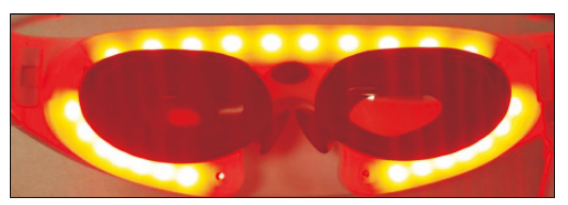

Patch contact pin

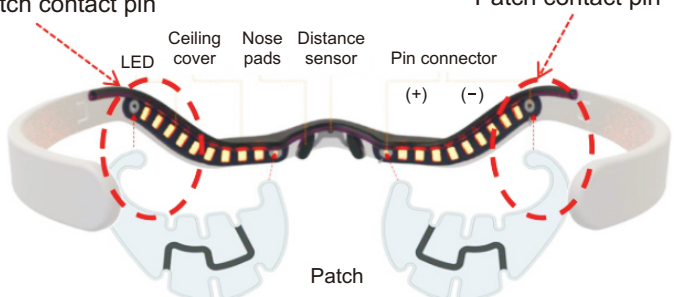

C

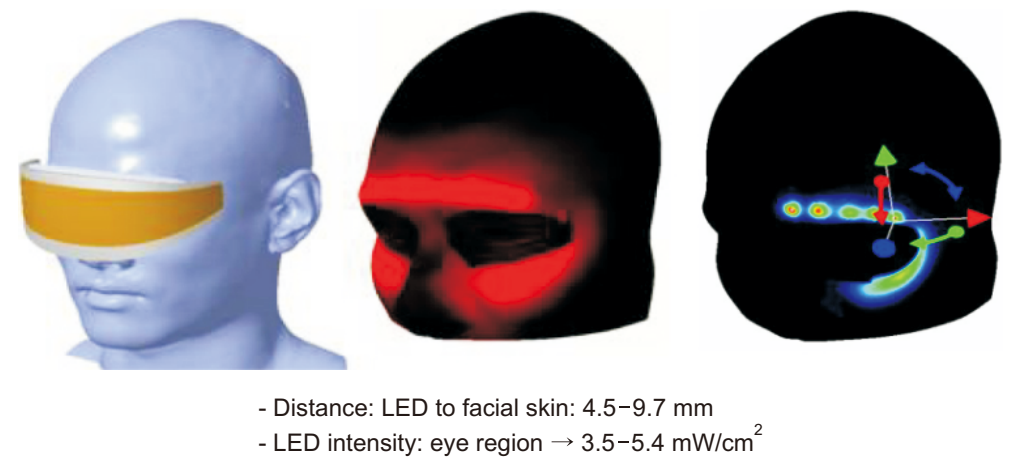

Fig. 1. The 630- and 850-nm lightemitting diode (LED) with microcurrent device for skin rejuvenation. (A, B) The EWN1 device (obtained from LG electronics Inc., with permission). (C) Performance evaluation of surface delivered energy for 630-nm and 850-nm LED and microcurrent treatment by computer aided engineering (CAE) measurement. 
adjacent areas was performed using a folliscope (LeedM, Seoul, Koreal (Data not shown). The skin of Yorkshire white pigs had similar absorption, allergic reaction, and epithelial structure to humans, making it suitable for dermal administration skin test. ${ }^{9}$ Since the skin has less body hair than other species, it is easy to use for skin test evaluation.

\section{Histological examination}

After treating the EWN1 device, hematoxyline and eosin (H\&E), Masson's trichrome (M\&T), and Victoria blue staining were performed for histopathological and cell survival rate evaluation in the skin tissue. After 14 days of treatment (week 2) and after 42 days (week 6), an 8 mm punch biopsy was performed to store the biopsied skin tissue. The tissue was fixed with $4 \%$ paraformaldehyde and embedded in paraffin. Next, 5 - $\mu$ m-thick sections were transferred to probe-on-plus slides (Fisher Scientific, Pittsburg, PA, USA) and stained with H\&E, M\&T, and Victoria blue.

\section{Measurement of collagen production in the skin}

Sircol soluble collagen assay kit (Biocolor, Belfast, UK) was used to measure the amount of collagen contained in the skin tissues of all groups. A kit was purchased, and a collagen assay was performed. The skin tissue had the same weight. Pepsin and 0.5M acetic acid 1:10 ratio of pepsin/tissue wet weight) were added, followed by overnight incubation at $4^{\circ} \mathrm{C}$. Subsequently, $100 \mu \mathrm{L}$ of Sircol dye reagent was added, followed by shaking for $30 \mathrm{~min}$ and shaking for $10 \mathrm{~min}$ at 12,000 rpm. After centrifugation, $750 \mu \mathrm{L}$ of acid-salt wash was added, and the mixture was washed with water followed by lightly mixing an alkali reagent in the microplate. This was measured at a wavelength of $555 \mathrm{~nm}$. For the collagen calibration standard curve, a reference point was set by adding $0,5,10$, and 15 $\mu \mathrm{L}$ of collagen standard solution $(0.5 \mathrm{mg} / \mathrm{mL})$ each.

\section{Measurement of the amount of elastin produced in the skin}

The effect on elastin synthesis was observed using the FASTIN elastin assay (Biocolor). Samples or standards were mixed in the same amount with the precipitation reagent ( $0.25 \mathrm{M}$ oxalic acid), left for $10 \mathrm{~min}$, and centrifuged at $10,000 \times \mathrm{g}$ for $10 \mathrm{~min}$. After centrifugation, the supernatant was removed; $1 \mathrm{~mL}$ of Fastin Dye Reagent was added, reacted for $90 \mathrm{~min}$, and then centrifuged at 10,000 $\times \mathrm{g}$ for $10 \mathrm{~min}$ to separate the elastin-dye complex. The supernatant was removed, $250 \mu \mathrm{L}$ of dye dissociation reagent was added, mixed, and absorbance was measured at $513 \mathrm{~nm}$ using an ELISA microplate reader. The elastin concentration was calculated after substituting into the $\alpha$ -elastin standard curve.

\section{Immunohistochemical analyses}

Immunohistochemical staining for collagen type I (Anti-Col1A1, NB600-408; Novus Biologicals, Littleton, CO, USA) involves deparaffining a $4.5 \mu \mathrm{m}$-thick paraffin section and washing with xylene, ethanol, distilled water, and the avidin-biotin peroxidase complex (ABC), in order. Immunohistochemical staining was performed using this method. The samples were transferred to a 3\% hydrogen peroxide solution, quenched at room temperature for 10 minutes, and washed 3 times with $0.1 \mathrm{M}$ tris-buffered saline (DAKO, Carpinteria, CA, USA). After that, they were transferred to $0.05 \%$ trypsin and allowed to stand at $37^{\circ} \mathrm{C}$ for $10 \mathrm{~min}$, washed three times with Tris-buffered saline, and allowed to stand at room temperature for $10 \mathrm{~min}$ in a protein blocking agent (Immunon, Pittsburgh, PA, USA) to saturate the non-specific protein binding site. After washing the sections with phosphate-buffered saline (PBS), they were incubated with the primary antigen overnight at $4^{\circ} \mathrm{C}$. After washing with PBS for 5 min, they were left in biotinylated universal secondary antibody reagent $(\mathrm{Im}$ munonl for $30 \mathrm{~min}$, and then washed with PBS for $10 \mathrm{~min}$. They were reacted with horseradish peroxidase at room temperature for $30 \mathrm{~min}$, washed three times with PBS for 5 min with care, and reacted with diaminobenzidine (DAB). Contrast staining with Mayer's hematoxylin (Sigma, St Louis, MO, USA) was observed under a microscope.

These corrected images were then imported into ImageJ software (NIH) (http://imagej.nih.gov/ij/) where a region of interest was defined by the epidermis and dermal layer. The percentage of black pixels was taken to represent the percentage of tissue within the skin section. ${ }^{10}$

\section{Statistical analysis}

All data are expressed as mean \pm standard deviation. For statistical analysis of each set of data, Student's t-test was performed. Values of $p<0.05$ were considered statistically significant.

\section{RESULTS}

\section{Instrument and histological evaluation}

To determine whether the skin was irritated by the EWN1 device, the skin conditions of the treated and untreated areas were observed. All evaluations were conducted immediately after irradiation (day 0 ) and before and after the device treatment for 6 weeks. During the 
test period, a visual evaluation was conducted by the research director. In all treatment groups, no adverse skin reactions, including epidermal damage, erythema including blisters, and heat damage reactions, were observed in the visual evaluation (Fig. 2A).

To observe the change in the skin surface condition according to the EWN1 device treatment, magnification was taken with a Folliscope (Data not shown). At different time points after skin treatment using the device during the period (treatment twice a week) according to each condition (immediately after irradiation [day 0], after 14 days [after 2 weeks, 4 times], and after 42 days [after 6 weeks, 12 times]), no difference in heat damage and erythema were observed on the skin surface in comparison with the untreated control group. Standard treatment protocols are for treatments of $15 \mathrm{~min}$ for a $10 \times 10 \mathrm{~cm}$ area, keeping the surface temperature at $33.3^{\circ} \mathrm{C}$ to $36.8^{\circ} \mathrm{C}$. Importantly, the combination treatment was much more effective in absorbing energy than the LED or microcurrent single treatments.

After the EWN1 device treatment, H\&E staining was performed for histopathological evaluation of the skin.
As shown in Fig. 2B, no abnormal infiltration or dermal necrosis of inflammatory cells was observed in any skin tissues of the no treatment, microcurrent only, LEDs only, LEDs/Microcurrent combination treatment, and no epidermal damage or adverse reactions in the dermis were observed.

\section{Combination of 630-and 850-nm LEDs/Microcur- rent induced collagen and elastin synthesis}

Collagen is the main component of the skin dermis, and it decreases with age, causing wrinkles in the skin. To confirm the formation of collagen in the skin tissue after EWN1 device treatment (Fig. 3B). As a result of collagen synthesis, at week 2 (use 4 times), there was a statistically significant increase in the LEDs group 12.31 -fold, $130.5 \%$ increase) and the LEDs/Microcurrent combination group (2.70-fold, $169.8 \%$ increase) compared to the no treatment group. In the $6^{\text {th }}$ week (use 12 times) result, compared to no treatment, the LEDs group (1.25-fold, $74.6 \%$ increased), the microcurrent alone group (1.68-fold, $68.0 \%$ increased), and the combined LEDs/Microcurrent group (2.25-fold, $124.6 \%$ increase), a statistically signifi-

A
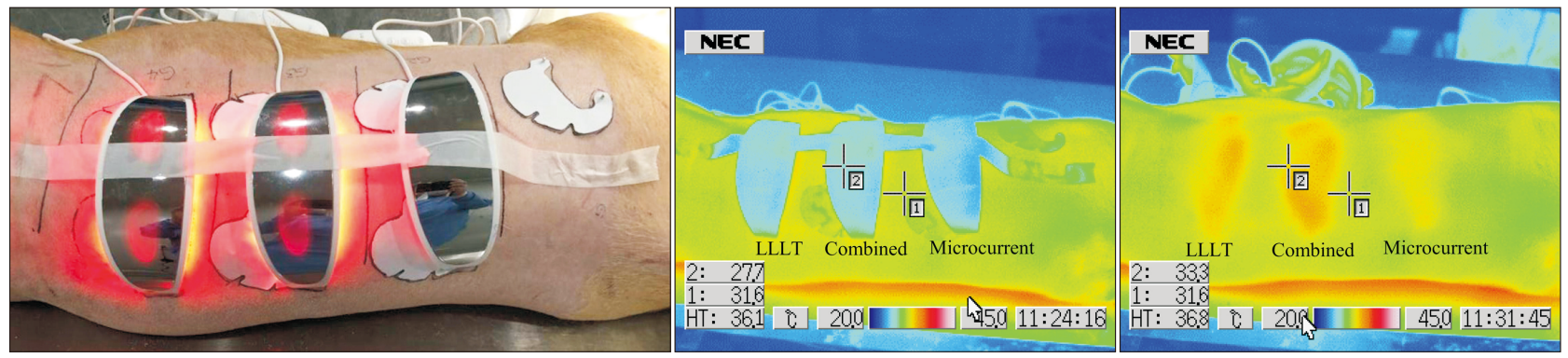

B

level

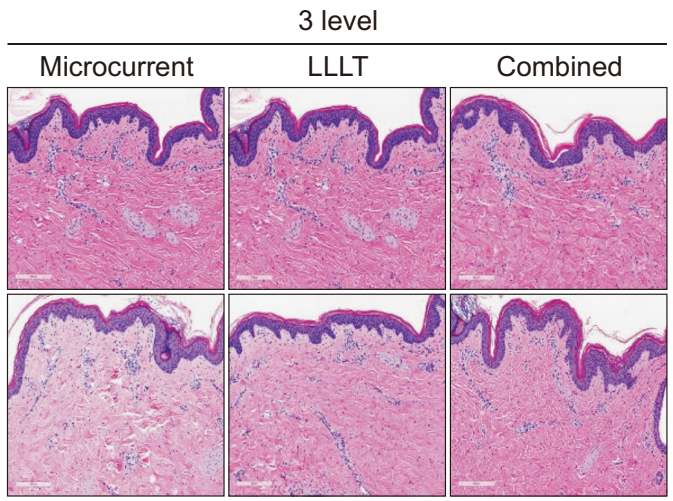

$(\times 100)$

Fig. 2. Thermal and histological changes after treatment. (A) Surface temperature changes of the porcine model during application of 630-nm and 850-nm LED and microcurrent and after device removal immediately after treatment. This device delivered heat did not affect surrounding skin tissue. (B) Tissue biopsy was done before 630-nm and 850-nm LED and microcurrent treatment (0), and on weeks 2, 6. The histological effect of the device on porcine dorsal skin was analyzed using hematoxylin-eosin $(H \& E)$ stain $(\times 100)$. Simultaneous samples are showing greater vascularization, denser organization, and thickness of connective fibers in comparison with the control. LLLT, low-level light therapy. 


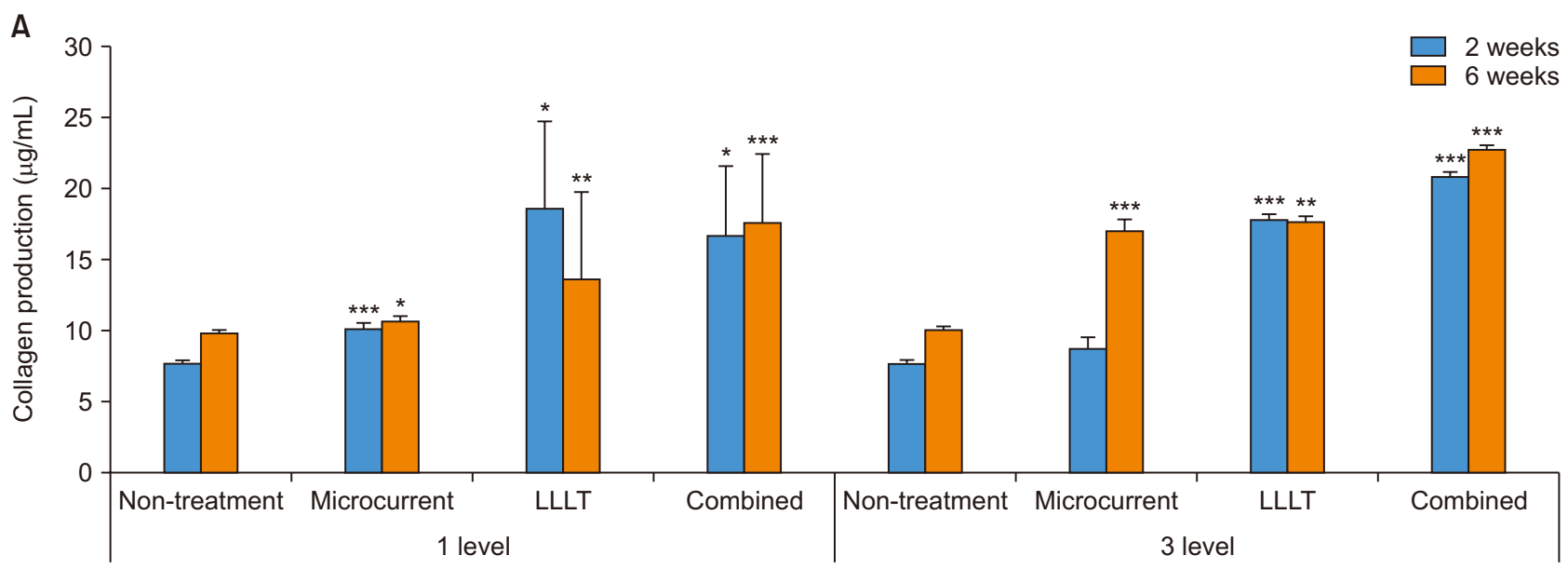

B
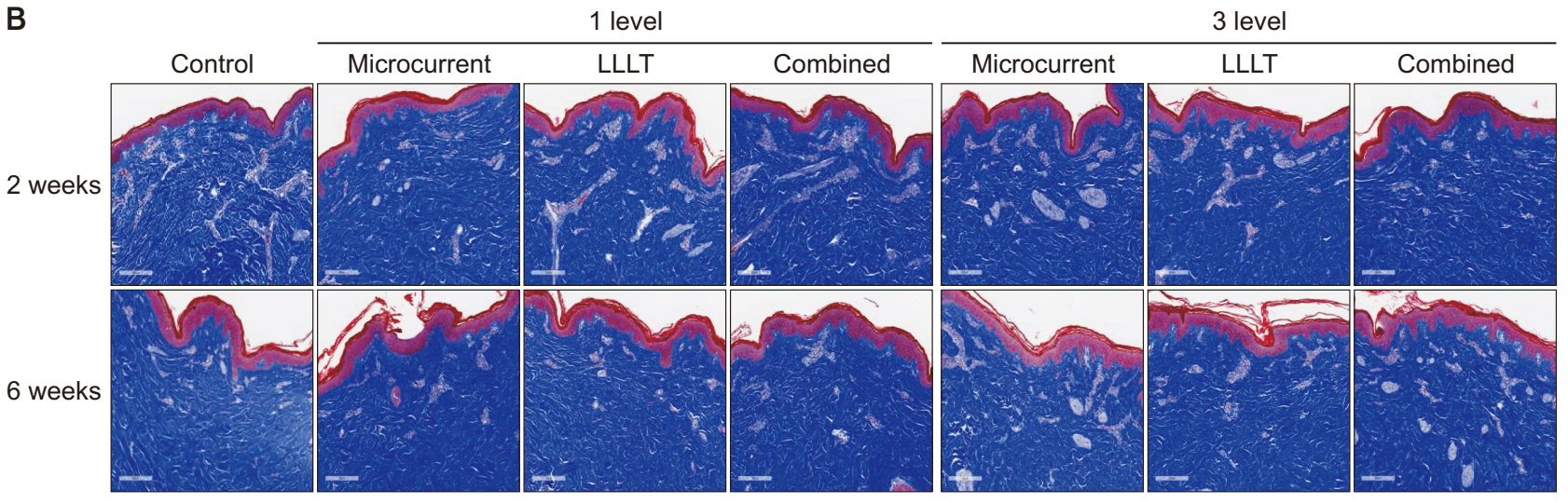

$(\times 100)$

Fig. 3. Change of collagen within porcine skin samples. (A) In addition tissue samples were solubilized and the Sircol collagen extracted and quantified in $\mu \mathrm{g} / \mathrm{mg}$ tissue \pm standard deviation (SD) by colorimetric assay. Statistically significant variations were indicated as $* p<0.05, * * p<$ $0.01,{ }^{* *} p<0.001$. (B) Skin samples were stained with Masson's trichrome. Collagen fibers were stained in blue, and pictures were taken at $\times 100$.

cant increase in all was confirmed (Fig. 3A).

In addition, as a result of the amount of elastin synthesis, statistically significant increases were observed in the microcurrent alone group (1.25-fold, 25.3\% increase) and LEDs group (1.72-fold, $71.8 \%$ increase) compared to no treatment at week 2 (use 4 times). In the $6^{\text {th }}$ week (12 uses), statistically significant increases were found in the microcurrent alone group (1.63-fold, 63.5\% increase) and the LEDs/Microcurrent combination group (1.92-fold, 91.9\% increase) compared to the no treatment group (Fig. 4A). The LEDs/Microcurrent used in this procedure collectively acts on the epidermis and dermis layer of the skin, inducing regeneration of collagen and elastic fibers through cell activation and a complex process such as synthesis and reconstruction of collagen fibers. These results strongly suggest that collagen and elastin biosynthesis is affected by the combination of LEDs/Microcurrent.

\section{The 630- and 850-nm LEDs/Microcurrent induc- es neocollagenesis and neoelastinogenesis}

Next, the change, loss, amount, and morphology of elastic fibers in the dermal layer of the skin were observed using Victoria blue staining (blue color; elastic fibers) (Fig. 4B). Overall, an increase in elastic fibers was seen both 2 and 6 weeks after treatment. As a result, it is thought that the LEDs/Microcurrent energy generated in EWN1 is evenly transmitted to the skin layer, affecting skin elasticity.

In this experiment, it was confirmed whether treatment with the EWN1 device has an effect on collagen synthesis in the dermis, and changes in the morphology of collagen fibers were observed in the process of tissue restoration after applying LEDs/Microcurrent. Upon observation with an optical microscope, the collagen fibers in all test groups were relatively denser and had more regular arrangement in the tissue after the device treatment compared to the untreated group (Fig. 5A). In particular, after 

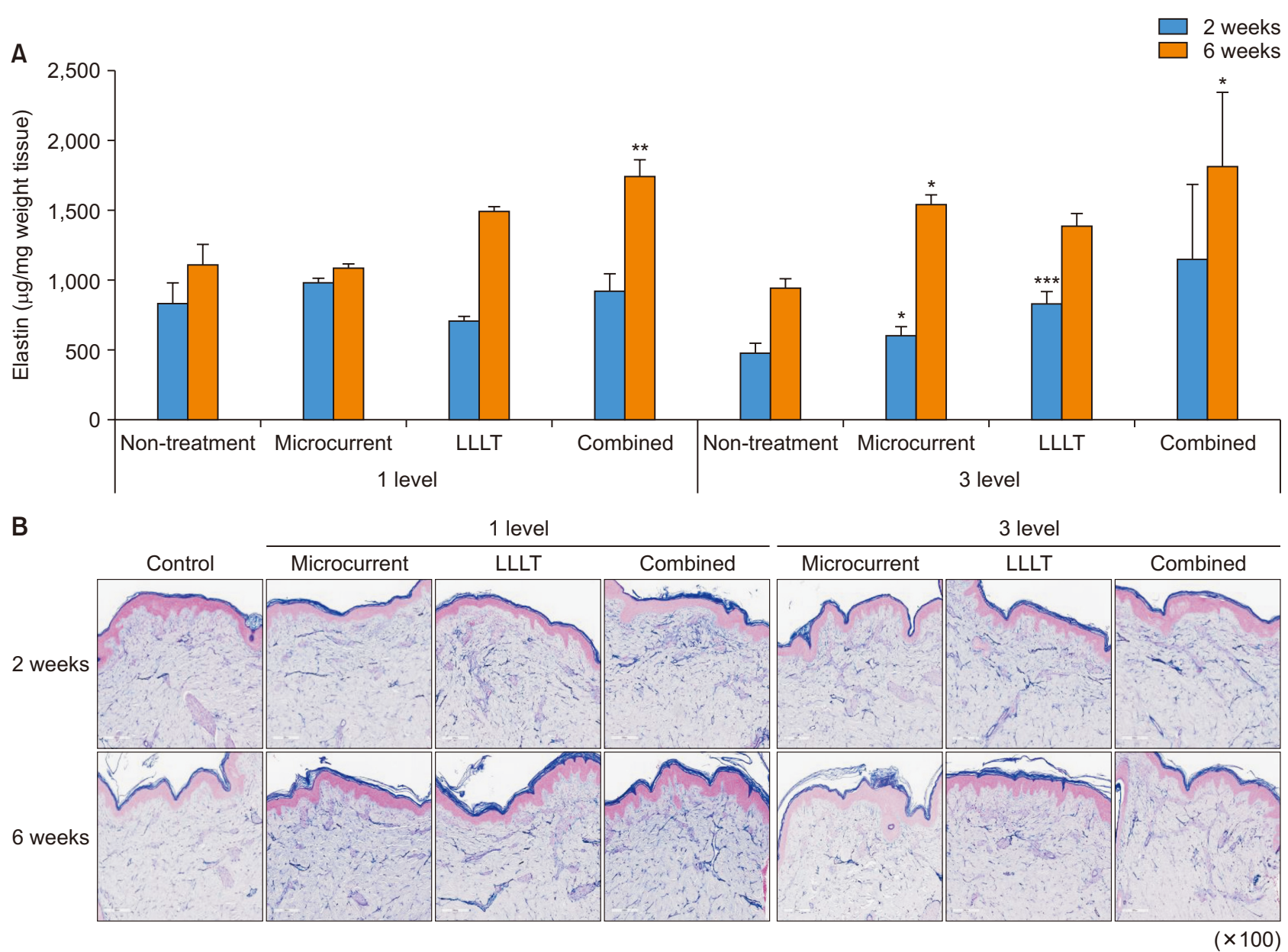

Fig. 4. Change of elastin within porcine skin samples. (A) In addition tissue samples were solubilized and the Elastin extracted and quantified in $\mu \mathrm{g} / \mathrm{mg}$ tissue $( \pm \mathrm{SD})$ by colorimetric assay. Statistically significant variations were indicated as $* p<0.05,{ }^{* *} p<0.01, * * * p<0.001$. (B) Skin samples were stained with Victoria blue. Elastin fibers were stained in blue, and pictures were taken at $\times 100$. The amount of elastin stained in blue significantly increased post-treatment compared with control.

treatment with the microcurrent, the change was evident in the upper part of the epidermis and dermis, and after treatment with LEDs, it was confirmed that there was an effect on the lower part of the dermis. As a result, it is believed that the combination treatment of LEDs/Microcurrent evenly affects the skin layers by transferring energy from the skin surface.

Also, type I collagen staining was performed by immunohistochemical staining, and the changes in collagen in the dermal layer of the skin were observed by EWN1 device treatment (Fig. 5B). LED technology is known to improve wrinkles by inducing collagen synthesis by selectively stimulating the production of collagen in the papillary dermis and upper reticular dermis without damaging the epidermis. Accordingly, the pattern of the collagen fibers was observed to be tighter in the treated groups than in the untreated group. Thus, at the $6^{\text {th }}$ week of use, an increase in collagen and elastic fibers was found at the top of the dermis in the skin of the test group.

\section{DISCUSSION}

The convergence of advanced technology improves the efficacy, ease of use, and safety of aesthetic devices. ${ }^{11}$ It is expected to continue to grow in the future in a social environment that places more importance on the convenience and well-being of each patient. A deeper understanding of intrinsic and extrinsic factors contributing to changes in the face. ${ }^{12}$

Although individualized combination therapy reflected modern esthetic practice in 2014 , nearly half of all cosmetic patients in the United States requesting noninvasive or minimally invasive interventions received multiple cosmetic procedures at the same time. ${ }^{13}$ A multifaceted 
A
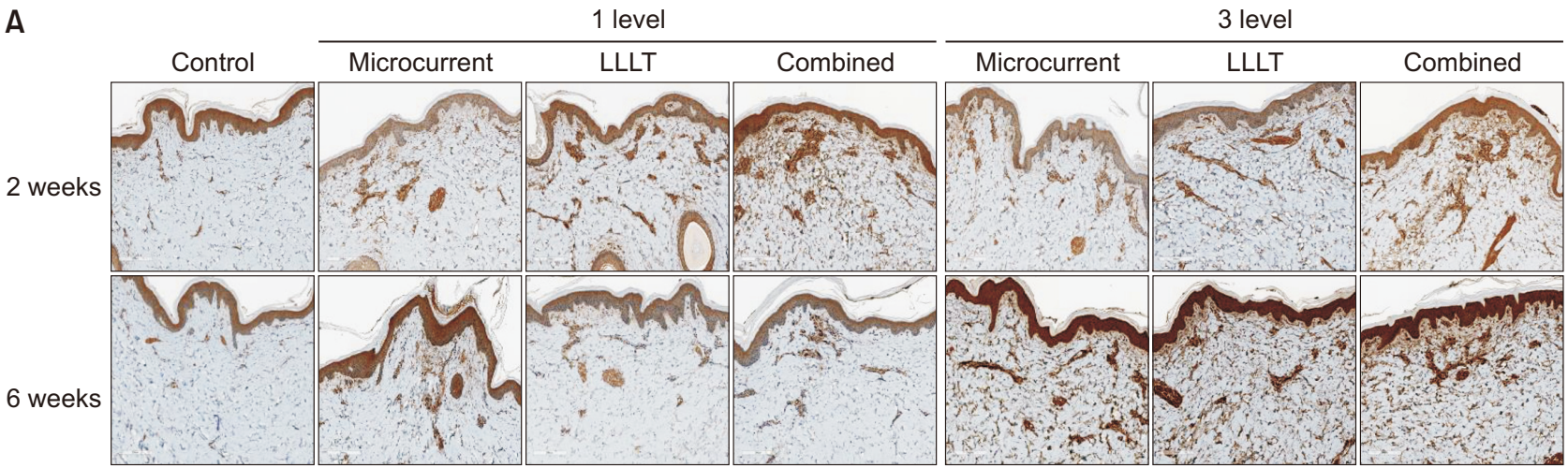

$(\times 100)$

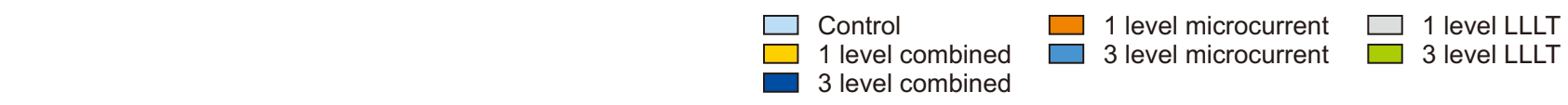

B

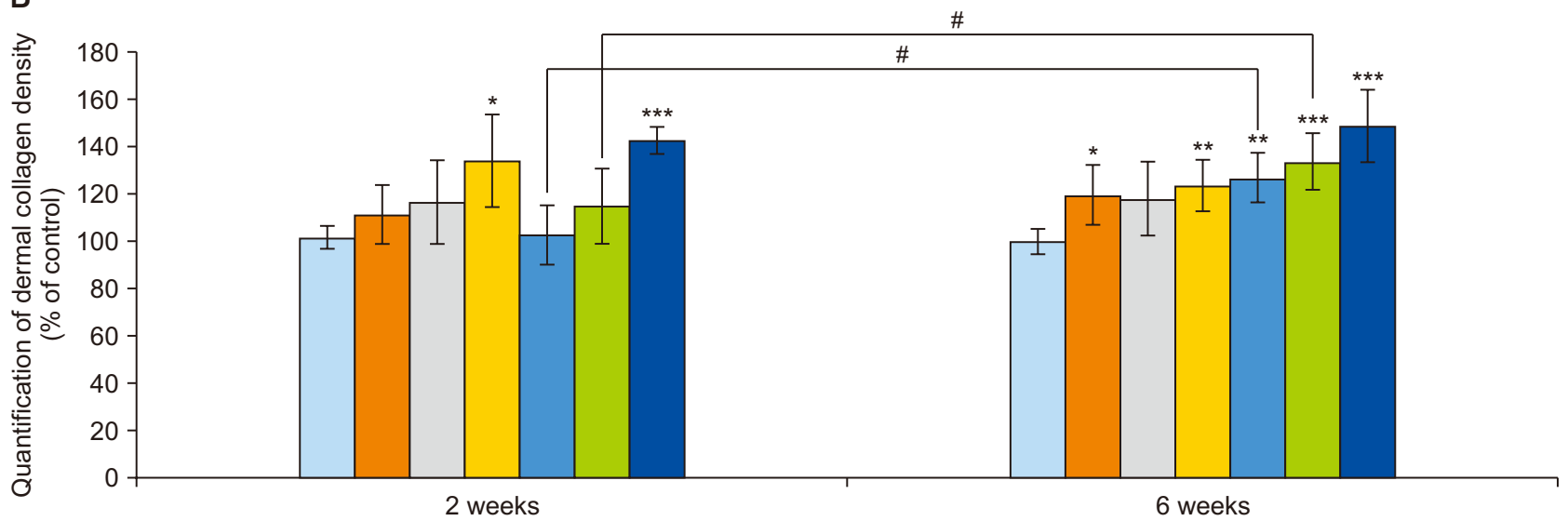

Fig. 5. Immunohistochemistry for COL1A1 in skin of porcine treated or untreated with 630-and 850-nm LED and microcurrent. Upregulated COL1A1 expression at protein levels strongly correlates with simultaneously 630-nm and 850-nm LED and microcurrent application. (A) Representative immunohistochemical photo-image (upper) and (B) graphical quantification (lower) of COL1A1 protein expression in each group. COL1A1 is highly expressed in 630-nm and 850-nm LED only and microcurrent only group compared to combined treatment. Statistically significant variations were indicated as ${ }^{*} p<0.05,{ }^{* *} p<0.01,{ }^{* * *} p<0.001$ (vs Control), ${ }^{\#} p<0.05$ (vs 2 weeks).

approach that safely incorporates two or more modalities-administered concomitantly or in the recommended sequence-is required along with an ongoing skincare regimen for optimal esthetic benefits. ${ }^{14,15}$ The combined application of these energies resulted in enhanced synergistic effects in several instances. ${ }^{16,17}$ In particular, a previous study reported the clinical experience of the lifting effect through the simultaneous investigation of HIFU and RF. ${ }^{18} \mathrm{HIFU}$ affects deep tissues and impacts the focal regions. Monopolar RF also affects deep tissues but impacts diffuse regions. We believe that these data provide further insights into effective skin tightening. Compared with the consecutive application of the modalities, the simultaneous use of these two fields has a more beneficial effect on animal dermal and subdermal tissues. This porcine model study indicates that the simultaneous applica- tion of LEDs/Microcurrent may be a suitable treatment for various skin conditions, such as rejuvenation.

The use of electricity, electrical stimulation, and electromagnetic fields is not new in medicine. Studies have shown that by externally applying an electric field or electric current, potentials present inside and between cells in soft tissues can facilitate biological and physiological changes in these tissues. In fact, there is strong experimental evidence suggesting that tendon repair can be significantly affected by electrical stimulation with intensity at the microcurrent level. ${ }^{19}$ Microcurrent is understood to be distinct from other forms of therapeutic electrical stimulation because the current intensity is much less than that of other forms of electrotherapy, such as percutaneous electrical nerve stimulation or paradic units. Microcurrent applications are believed to be effective in 
affecting and modifying cellular processes and activities. ${ }^{20}$ The use of different levels of current, frequency, and polarity has been shown to have various effects on different cell groups.

Low-intensity phototherapy, commonly referred to as "photobiomodulation," modulates numerous cellular functions by light in the far-infrared to near-infrared (NIR) regions of the spectrum (630-1000 nm). ${ }^{21}$ At the cellular level, photobiological regulation can stimulate macrophages and lymphocytes by regulating fibroblast proliferation and adhesion and synthesis of collagen and procollagen, promoting angiogenesis and improving energy metabolism within the mitochondria. ${ }^{22}$ In addition, photobiomodulation has been demonstrated to promote the production of growth factors such as keratinocyte growth factor (KGF), transforming growth factor (TGF), and platelet-derived growth factor (PDGF). However, our study had several limitations. Further study is needed to confirm and elucidate the reasons for these growth factors.

Previous findings were able to correlate fibroblast activity with the process of dermal matrix remodeling, with increased intradermal collagen density and decreased signs of aging. The proposed underlying mechanisms include photostimulation of terminal molecules in the electron transport chain and subsequent increase in adenosine triphosphate (ATP) concentration, selective photodriven activation of water molecules to enhance metabolic exchange and affect the ion transport systems found in the cell membrane. Detailed analysis of the gene expression profile of human fibroblasts showed the effect of low-intensity red light with a wavelength of $628 \mathrm{~nm}$ on 111 different genes involved in cellular functions such as cell proliferation; apoptosis; stress response; protein, lipid, and carbohydrate metabolism; mitochondrial energy metabolism; DNA synthesis and repair; and antioxidantrelated functions related to the cytoskeleton and cell-cell interactions. ${ }^{23}$ The specific role of reactive oxygen species (ROS) in increasing fibroblast proliferation and motility has recently been reported; thus, the elevation of ROS through photodynamic therapy can lead to a mitogenactivated protein kinase (MAPK) signaling pathway in skin fibroblasts, suggesting that ROS can improve cellular function in vitro.

Simultaneously, LEDs/Microcurrent application can be a useful tool to assess the induction of collagen and elastin remodeling in porcine skin. There is not much difference in the mechanism between LEDs- and microcurrent-induced cell activation, such as ATP synthesis, but synergistic effects are strongly suggested when using the technologies in combination because the actual application areas on the skin are different. Therefore, we evaluated the safety and effectiveness of the EWN1 device for the skin. Damage to the skin surface, abnormal inflammatory reactions and laceration reactions of the dermis, and tissue necrosis by the device treatment were not observed. Quantitative analysis of the amount of collagen and elastin produced by biopsy of skin tissue showed a statistically significant increase. Observing the change, loss, amount, and shape of the elastic fibers in the skin's dermal layer under each test condition (microcurrent only, LEDs only, and LEDs/Microcurrent combination), it was confirmed that the regeneration of collagen and elastic fibers was induced in the entire upper and lower layers of the dermis as energy was transferred well from each modality. The LEDs/Microcurrent combination has not yet been studied in a porcine model. In the future, additional studies should be performed to determine the safety and effectiveness of collagen and elastin stimulation in clinical study.

In this study, the effect of the EWN1 device on the production of collagen and elastin in the skin as well as the skin safety of the device was confirmed. However, a limitation was also observed in the amount of collagen and elastin production in the same tissue after 12 times uses compared to four uses, and additional research on the number of uses and long-term use is needed. Simultaneous LEDs/Microcurrent application is, at minimum, noninferior to individual LED and microcurrent application in terms of collagen and elastin induction. Nevertheless, it has the advantages of less pain, downtime, and frequency of side effects.

These results indicate that simultaneous application of 630-nm and 850-nm LED and microcurrent is an excellent choice for facial skin rejuvenation, indicating the synergistic action of these two modalities.

\section{ACKNOWLEDGMENTS}

We thank CRONEX (Hwasung, Korea) for its support in this study.

\section{CONFLICT OF INTEREST}

No potential conflict of interest relevant to this article was reported.

\section{FUNDING}

None. 


\section{REFERENCES}

1. Benedetto AV. What's new in cosmetic dermatology. Dermatol Clin 2019;37:117-28.

2. Clarke LH, Korotchenko A. Aging and the body: a review. Can J Aging 2011;30:495-510.

3. Wang B. Photoaging: a review of current concepts of pathogenesis. J Cutan Med Surg 2011;15 Suppl 1:S374-7.

4. Barolet D, Roberge CJ, Auger FA, Boucher A, Germain L. Regulation of skin collagen metabolism in vitro using a pulsed 660 $\mathrm{nm}$ LED light source: clinical correlation with a single-blinded study. J Invest Dermatol 2009;129:2751-9.

5. Li WH, Seo I, Kim B, Fassih A, Southall MD, Parsa R. Low-level red plus near infrared lights combination induces expressions of collagen and elastin in human skin in vitro. Int J Cosmet Sci. In press 2021.

6. Smith RB. Microcurrent therapies: emerging theories of physiological information processing. NeuroRehabilitation 2002;17:3-7.

7. He B. Bioelectricity of living tissue. IEEE Eng Med Biol Mag 1998;17:72, 117.

8. Gold MH, Biron J, Levi L, Sensing W. Safety, efficacy, and usage compliance of home-use device utilizing RF and light energies for treating periorbital wrinkles. J Cosmet Dermatol 2017; 16:95-102.

9. Wei JCJ, Edwards GA, Martin DJ, Huang H, Crichton ML, Kendall MAF. Allometric scaling of skin thickness, elasticity, viscoelasticity to mass for micro-medical device translation: from mice, rats, rabbits, pigs to humans. Sci Rep 2017;7:15885.

10. Schneider CA, Rasband WS, Eliceiri KW. NIH Image to ImageJ: 25 years of image analysis. Nat Methods 2012;9:671-5.

11. Wang JV, Saedi N, Geronemus RG. Differentiation in a market of imitation: the evolving world of aesthetic dermatology. J Cosmet Dermatol 2020;19:2987-9.

12. Sherber NS, Rad AN. Future directions in facial rejuvenation. Facial Plast Surg 2014;30:72-5.

13. Humphrey S, Beleznay K, Fitzgerald R. Combination therapy in midfacial rejuvenation. Dermatol Surg 2016;42 Suppl 2:S83-8.

14. Nestor MS. Combination therapy in clinical and cosmetic dermatology: the marriage of device and drug. J Drugs Dermatol 2004;3(5 Suppl):S4-11.
15. Coleman KM, Pozner J. Combination therapy for rejuvenation of the outer thigh and buttock: a review and our experience. Dermatol Surg 2016:42 Suppl 2:S124-30.

16. Collawn SS. Combination therapy: utilization of $\mathrm{CO} 2$ and Erbium:YAG lasers for skin resurfacing. Ann Plast Surg 1999;42:21-6.

17. Omi T, Kawana S, Sato S, Naito Z. Histological evidence for skin rejuvenation using a combination of pneumatic energy, broadband light, and growth factor therapy. J Cosmet Laser Ther 2010;12:222-6.

18. Shek SY, Yeung CK, Chan JC, Chan HH. The efficacy of a combination non-thermal focused ultrasound and radiofrequency device for noninvasive body contouring in Asians. Lasers Surg Med 2016;48:203-7.

19. Lin $\mathrm{YL}$, Moolenaar $\mathrm{H}$, van Weeren PR, van de Lest $\mathrm{CH}$. Effect of microcurrent electrical tissue stimulation on equine tenocytes in culture. Am J Vet Res 2006;67:271-6.

20. Poltawski L, Johnson M, Watson T. Microcurrent therapy in the management of chronic tennis elbow: pilot studies to optimize parameters. Physiother Res Int 2012;17:157-66.

21. Hamblin MR. Mechanisms and mitochondrial redox signaling in photobiomodulation. Photochem Photobiol 2018;94:199-212.

22. Tatmatsu-Rocha JC, Tim CR, Avo L, Bernardes-Filho R, Brassolatti P. Kido HW, et al. Mitochondrial dynamics (fission and fusionl and collagen production in a rat model of diabetic wound healing treated by photobiomodulation: comparison of $904 \mathrm{~nm}$ laser and $850 \mathrm{~nm}$ light-emitting diode (LED). J Photochem Photobiol B 2018;187:41-7.

23. Zhang Y, Song S, Fong CC, Tsang CH, Yang Z, Yang M. cDNA microarray analysis of gene expression profiles in human fibroblast cells irradiated with red light. J Invest Dermatol 2003;120:849-57.

How to cite this article: Kwon TR, Moon DW, Kim J, Kim HJ, Lee SJ, Han Y, Dan HW, Chi SH, Seong HM, Kim HJ, Lim GS, Lee J. Application of 630-nm and 850-nm light-emitting diodes and microcurrent to accelerate collagen and elastin deposition in porcine skin. Med Lasers 2021;10:96-105. https://doi. org/10.25289/ML.2021.10.2.96 